\title{
Alterations in head shape of newborn infants after caesarean section or vaginal delivery
}

\author{
S. W. DE SOUZA, J. ROSS, and R. D. G. MILNER \\ From the Department of Child Health, University of Manchester, St. Mary's Hospital
}

\begin{abstract}
De Souza, S. W., Ross, J. and Milner, R. D. G. (1976). Archives of Disease in Childhood, 51, 624. Alterations in head shape of newborn infants after caesarean section or vaginal delivery. Alterations of head shape in preterm, small-for-dates, and term normal infants were studied by measuring occipitofrontal circumference (OFC), biparietal diameter (BPD), and occipitofrontal diameter (OFD) at intervals after birth. In 9 preterm infants born by elective caesarean section there was a $5 \cdot 2 \%$ reduction in BPD and $2.0 \%$ reduction in OFC at the age of 7 days. In 18 term infants born by elective caesarean section these changes were $2.4 \%$ and $0 \%$ respectively in BPD and OFC. In 25 preterm infants born by vertex vaginal delivery there was a significant fall in OFC of $0.7 \%$ at the age of 7 days and of $2.4 \%$ in BPD, but no significant change in OFD. In 19 small-for-dates infants born vaginally OFC increased $1.0 \%$ and OFD $2.7 \%$ at 7 days, but BPD decreased $2.5 \%$. After the first week all three measurements increased in both groups of vaginal deliveries. The results show that shrinkage and biparietal flattening of the skull occur during the first week of life in preterm and term infants born by caesarean section and in preterm infants born vaginally. This fact should be borne in mind when comparing the measurements of an infant's head size with published norms.
\end{abstract}

The occipitofrontal circumference (OFC) is used as a measure of head size and in assessing postnatal head growth in newborn infants (Low, 1950; Thomson, 1956; Westropp and Barber, 1956; Falkner, 1958; Lubchenco, Hansman, and Boyd, 1966; Nellhaus, 1968). In such infants alterations in OFC may also take place when the shape of the head changes from a near circular to an elliptical one (Baum and Searls, 1971a, b). This change in shape may be due to moulding since the skull bones are separate and the skull is not rigid. The head may be moulded as it passes through the birth canal or after birth, by gravity, as the infant lies on one side or the other. It has been reported (Durkan and Russo, 1966; Willocks et al., 1964) that the steady increase in fetal biparietal diameter (BPD) during the third trimester of pregnancy is followed after birth by a sudden fall which may be due to moulding. Alterations in head shape after birth have been investigated in the present study in preterm infants, small-fordates infants born at term, and normal term infants.

Received 11 December 1975.

\section{Subjects and methods}

Infants. The infants studied were singletons of both sexes, born at St. Mary's Hospital during 1970-71. Some mothers were delivered by elective caesarean section and others by vertex vaginal delivery. Gestational age was calculated from the first day of the last menstrual period. Preterm infants were less than 37 weeks' gestational age. Term infants included those born between 37 and 42 weeks' gestational age. Term infants were defined as being small-for-dates if the birthweight was below the 10th centile (Butler and Alberman, 1969).

Measurement of head size. Measurements were made within 3 hours after birth and subsequently at varying intervals. OFCs were measured using a plastic measuring tape which was placed immediately above the supraorbital margins and horizontally round the most prominent part of the occiput. BPDs and occipitofrontal diameters (OFDs) were measured with a pair of obstetric calipers (Allen and Hanbury Ltd.) and a vernier rule (Mitutoyo dial calipers 505-603). The BPD measured was the maximum distance between the parietal eminences in the horizontal plane, and the OFD was the distance between the most prominent part of the occiput and the glabella. All measurements 
were made by one of us (J.R.) with the exception of one or two made during the first 24 hours in some of the infants born by caesarean section. Bach measurement was made three times and the maximum range of observations was $3 \mathrm{~mm}$.

Analysis of results. Results are presented as mean $\pm S E$ or range. The paired comparisons ' $t$ ' test was used when comparing two sets of results.

\section{Results}

Infants born by caesarean section. 9 preterm and 18 term infants were born by elective caesarean section. OFC and BPD measurements were made between birth and 3 hours of age, during the following 24 hours at 3- or 6-hourly intervals, and then at 24-hourly intervals for a total of 7 days postnatally.

In the preterm group OFCs and BPDs were measured in all infants within the first 3 hours after birth and subsequently 5-9 OFC and 6-9 BPD measurements were made at 3,6 , or 24-hourly intervals; $B P D$ and $O F C$ values were (mean $\pm S E$ ) $8 \cdot 59 \pm 0 \cdot 20$, and $31 \cdot 51 \pm 0 \cdot 42 \mathrm{~cm}(\mathrm{n}=9)$ respectively in the first 3 hours after birth. Percentage changes from the initial observations at each interval are shown in Fig. 1. A progressive fall in BPD and OFC occurred until day 3; mean BPD fell by $5 \cdot 2 \%(\mathrm{P}<0.025, \mathrm{n}=8)$ and mean OFC by $2.0 \%(P<0.02, n=8)$, after which they remained relatively steady. On day 7 there was a greater reduction in BPD than OFC (BPD 8.09 $\pm 0.19 \mathrm{~cm}$;

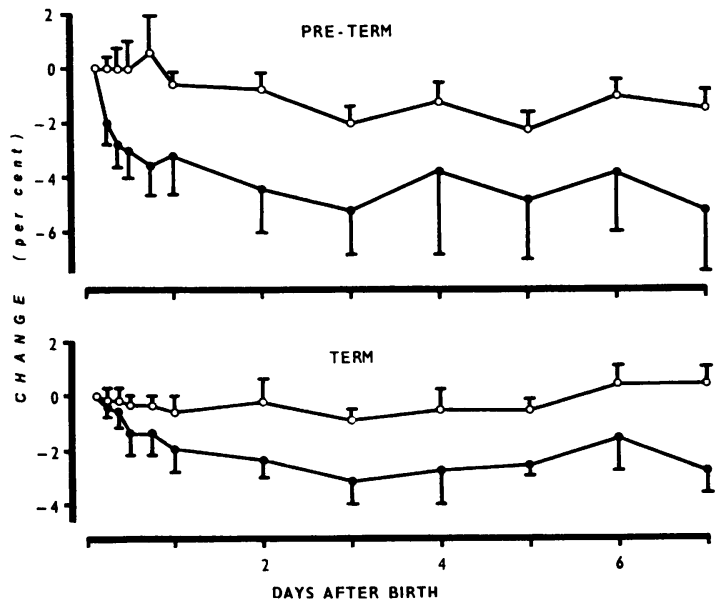

FIG. 1.-Percentage changes during the first 7 days after birth in BPD (O) and OFC (O) measurements in preterm infants and in term infants born by elective caesarean section. Mean \pm SE of 5-9 OFC and 6-9 BPD measurements in the preterm, 11-18 OFC and 12-18 BPD measurements in term infants are shown.
OFC $31 \cdot 03 \pm 0.42 \mathrm{~cm}, \mathrm{n}=9$ ) indicating that the heads had both shrunk and flattened in the biparietal diameter.

In the group born at term (Fig. 1) BPD and OFC values were $9 \cdot 36 \pm 0 \cdot 20$ and $34 \cdot 26 \pm 0.53 \mathrm{~cm}$ $(\mathrm{n}=8)$ respectively, and during the first 3 days there was a $2.4 \%$ fall in mean BPD ( $P<0.001$, $\mathrm{n}=17$ ) which then remained relatively steady being $9 \cdot 11 \pm 0.87 \mathrm{~cm}(\mathrm{n}=18)$ on day 7 . In contrast, mean OFC fell $1 \cdot 1 \%(P<0 \cdot 05, n=13)$ on day 3, remained steady for a further 2 days, and then gradually increased to $34 \cdot 40 \pm 0.62 \mathrm{~cm}$ on day 7 . By the end of the first week these infants had regained the head circumference seen at birth but showed a small degree of biparietal flattening.

Infants born by vertex vaginal delivery. OFC, OFD, and BPD were measured within the first 3 hours after birth and then at 7- to 8-day intervals for 28-29 days after birth. 25 preterm (26-35 weeks' gestational age) and 19 small-fordates infants born at term were studied. Birthweights in the two groups were similar. In the preterm group the initial mean (and range) OFD, OFC, and BPD values of 25 infants were 10.09 $(9 \cdot 27-11 \cdot 26), 29 \cdot 1 \quad(24 \cdot 81-31 \cdot 58)$, and $7 \cdot 77$ $(6 \cdot 19-8 \cdot 48) \mathrm{cm}$ respectively. At each 7 - to 8-day interval after birth, 17-22 observations were made and the percentage changes from the initial observations are shown in Fig. 2 . 7 to 8 days after birth there was no change in OFD but mean OFC fell by $0.7 \%(\mathrm{P}<0.01, \mathrm{n}=22)$ and mean $\mathrm{BPD}$ $2 \cdot 4 \%(P<0 \cdot 001, n=22)$. Subsequently there was an increase in all 3 parameters, greatest in OFD

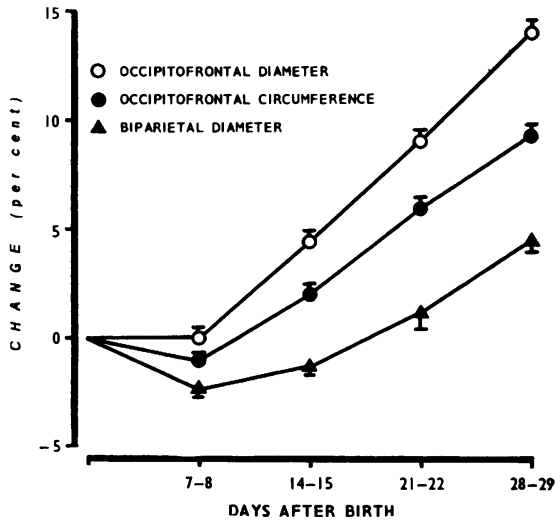

FIG. 2.-Percentage changes during the first 28-29 days after birth in BPD ( $\triangle), O F C$ (O), and OFD (O) in preterm infants of 26-35 weeks' gestational age born by vertex vaginal delivery. Mean $\pm S E$ of 17-22 observations at each 7- to 8-day interval is shown. 
and least in BPD. Initial OFC was regained on average in 9-10 days and initial BPD in 17-18 days.

$O F D, O F C$, and BPD values in the small-fordates group were $10 \cdot 59 \pm 0 \cdot 13,31 \cdot 07 \pm 0 \cdot 37$, and $8 \cdot 37 \pm 0 \cdot 11 \mathrm{~cm}(\mathrm{n}=19)$ respectively within 3 hours after birth. At 7- to 8-day intervals, 13-18 observations were made and the percentage change at each interval is shown in Fig. 3. 7 to 8 days after

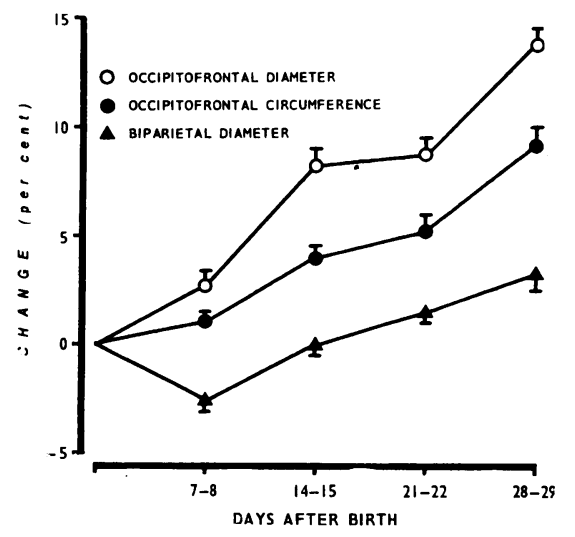

FIG. 3.-Percentage changes during the first 28-29 days after birth in $B P D(\Delta), O F C(O)$, or $O F D(O)$ in smallfor-dates term infants born by vertex vaginal delivery. Mean $\pm S E$ of 13-18 observations at each 7- to 8-day interval is shown.

birth there was a $2 \cdot 7 \%$ increase in OFD $(\mathrm{P}<0.001$, $\mathrm{n}=18)$ and a $1 \%$ increase in OFC $(\mathrm{P}<0.05$, $\mathrm{n}=18$ ) associated with a $2.5 \%$ decrease in BPD $(\mathrm{P}<0.001, \mathrm{n}=18)$. Subsequently there was a steady increase in all 3 parameters. Initial BPD was regained on average in 14-15 days. The rates of increase in the 3 parameters differed in the same manner as in preterm infants.

\section{Discussion}

OFC is a well established measurement of head size and charts relating OFC to age are used to assess head growth. Since centile lines on these charts have been derived from OFC values obtained by cross-sectional studies of children in a given population, the impression one may have is that OFC should increase along a certain growth trajectory. Nevertheless, the present study has shown that there is head shrinkage and biparietal flattening during the first week after birth. This fact should be borne in mind when comparing an infant's OFC with published normal data.

It has been suggested (Baum and Searls, 1971b) that the head of a fetus being surrounded by fluid develops with a near circular cross-section. During the first week after birth in infants of all gestational ages born by caesarean section and in preterm infants born vaginally there is shrinkage of the head and biparietal flattening. The reduction in OFC was unexpected since the biparietal flattening leads to an accentuation of the elliptical shape of the head. The greater biparietal flattening and reduction in head size in preterm infants by caesarean section compared with those born by vaginal delivery suggests that some shrinkage and biparietal flattening may have taken place during birth in the latter group of infants.

The deformity of head shape of preterm infants is greater than that of those born at term; this confirms a common clinical observation. Biparietal flattening postnatally is probably brought about by gravity as infants are nursed on one side or the other. By the same argument the rate of increase of BPD after the first week is least and that of OFD greatest (Fig. 2). A reduction in OFC also observed during the same period implies a reduction in head size which may be related to loss of body water (Brans et al., 1974).

Head shape differs in preterm compared with small-for-dates newborn infants. Alterations in OFD, OFC, or BPD in both groups born by vertex vaginal delivery are best seen during the first 7 days after birth. There was a significant fall of $0.7 \%$ in OFC and of $2.4 \%$ in BPD but no significant change in OFD, in preterm infants. By contrast, in small-for-dates infants the OFC increased $1.0 \%$ and the OFD $2 \cdot 7 \%$, but the BPD decreased $2.5 \%$. These differences are noteworthy especially since the two groups had similar birthweights. It is possible that such differences in head shape may persist in adult life (Baum and Searls, 1971b).

\section{REFERENCES}

Baum, J. D., and Searls, D. (1971a). Head shape and size of newborn infants. Developmental Medicine and Child Neurology, $13,572$.

Baum, J. D., and Searls, D. (1971b). Head shape and size of pre-term low-birthweight infants. Developmental Medicine and Child Neurology, 13, 576.

Brans, Y. W., Sumners, J. E., Dweck, H. S., and Cassady, G. (1974). A noninvasive approach to body composition in the neonate: dynamic skinfold measurements. Pediatric Research, 8, 215.

Butler, N. R., and Alberman, E. D. (1969). Maternal factors affecting duration of growth pregnancy, birthweight and foetal growth. Perinatal Problems, p. 47. Ed. by N. R. Butler and E. D. Alberman. Livingstone, Edinburgh and London.

Durkan, J. P., and Russo, G. L. (1966). Ultrasonic fetal cephalometry: accuracy, limitations and applications. Obstetrics and Gynecology, 27, 399.

Falkner, F. (1958). Some physical measurements in the first three years of life. Archives of Disease in Childhood, 33, 1.

Low, A. (1950). Measurements of infants at birth. Annals of Eugenics, 15, 210.

Lubchenco, L. O., Hausman, C., and Boyd, E. (1966). Intrauterine growth in length and head circumference as estimated from live births at gestational ages from 26 to 42 weeks. Pediatrics, 37, 403. 
Nellhaus, G. (1968). Head circumference from birth to eighteen years. Pediatrics, 41, 106.

Thomson, J. (1956). Infant growth. Archives of Disease in Childhood, 31, 382.

Westropp, C. K., and Barber, C. R. (1956). Growth of the skull in young children. Part I: standards of head circumference. fournal of Neurology, Neurosurgery and Psychiatry, 19, 52.
Willocks, J., Donald, I., Duggan, T. C., and Day, N. (1964). Foetal cephalometry by ultrasound. Fourna! of Obstetrics and Gynaecology of the British Commonwealth, 71, 11.

Correspondence to Dr. S. W. De Souza, Department of Child Health, St. Mary's Hospital, Manchester M13 OJH.

The following articles will appear in future issue of this journal:

Role of chronic hepatitis in development of thalassaemic liver disease. G. Masera, G. Jean, G. Gazzola, and M. Novakova.

Osmolar relation between cerebrospinal fluid and serum in hyperosmolar hypernatraemic dehydration. $A . H$. Habel and $H$. Simpson.

Origin of intraventricular haemorrhage in the preterm infant. G. Hambleton and J. S. Wigglesworth.

Arterial oxygen tension and response to oxygen breathing in differential diagnosis of congenital heart disease in infancy. R. W. A. Jones, J. H. Baumer, M. C. Joseph, and E. A. Shinebourne.

Prolonged rupture of membranes, pre-eclamptic toxaemia, and respiratory distress syndrome. M. L. Chiswick. Intrathecal antibiotic therapy for neonatal meningitis. C. Y. Yeung.

Multifocal osteolysis with nephropathy. R. Counahan, M. J. Simmons, and G. J. Charlwood.

'Juvenile' myasthenia gravis in early infancy. F. Oberklaid and I. J. Hopkins. 